\title{
Exclusive Hadronic Processes and Color Transparency
}

\section{Pankaj Jain $^{a}$ and John P. Ralston ${ }^{b}$}

\author{
${ }^{a}$ Physics Department, I.I.T. Kanpur, Kanpur - 208016 \\ ${ }^{b}$ Department of Physics and Astronomy, University of Kansas, \\ Lawrence, KS 66045 USA
}

\begin{abstract}
We review the current status of high energy exclusive processes and color transparency.
\end{abstract}

\section{Introduction}

It is known that at asymptotically large momentum transfer certain exclusive hadronic reactions are calculable within the framework of perturbative QCD (pQCD) due to asymptotic freedom. However the applicability of pQCD to exclusive processes remains controversial at the largest momentum transfers probed in the laboratory. The quark-counting scaling laws of Brodsky and Farrar [1] tend to agree remarkably well with data. This apparently indicates that a finite, minimal number of quarks are being probed. However, the helicity conservation selection rules of Lepage and Brodsky tend not to agree with data [1, 2, 3]. This indicates that the Brodsky-Lepage factorization scheme fails, independent of further details. Furthermore the formalism fails to correctly predict the magnitude of the proton electromagnetic form factor [ [4, 5, 6]. This suggests that the basic assumption in this formalism, namely that these processes are dominated by short distance, may not be true at laboratory energies. As a consequence, the agreement of the scaling laws with data becomes rather mysterious.

Compared to exclusive processes in free space, it has been shown [7, 8, 9] that the corresponding processes in a nuclear medium will be theoretically cleaner. Large quark separations will tend not to propagate in the strongly interacting nuclear medium. Configurations of small quark separations, on the other hand, which coincide with the perturbatively calculable region, will propagate with small attenuation. This phenomenon, called nuclear filtering [7, 8, 9], is the complement of the idea called color transparency [10]. 
In its original rendition, color transparency [10 was based on having large momentum transfer $Q^{2}$ select short distance, then free to propagate easily through a passive nuclear probe. Nuclear filtering uses the nuclear medium in an active way toward the same purpose, and may be more efffective.

\section{Exclusive Processes in Free Space}

Let us briefly review the framework for calculation of exclusive hadronic processes within pQCD. We take the pion electromagnetic form factor as an example. The short-distance formalism crucially depends on the assumption that the process can be factorized in a perturbatively calculable hard scattering piece and the soft distribution amplitude. Given this assumption, the pion electromagnetic form factor [2, 11, 12] at momentum transfer $q^{2}=-Q^{2}$ can be written as

$$
F_{\pi}\left(Q^{2}\right)=\int d x_{1} d x_{2} \phi\left(x_{2}, Q\right) H\left(x_{1}, x_{2}, Q\right) \phi\left(x_{1}, Q\right),
$$

where $\phi(x, Q)$ are the distribution amplitudes which can be expressed in terms of the pion wave function $\psi\left(x, \vec{k}_{T}\right)$ as

$$
\phi(x, Q)=\int^{Q} d^{2} k_{T} \psi\left(x, \vec{k}_{T}\right)
$$

Here $x$ is the longitudinal momentum fraction and $\vec{k}_{T}$ the transverse momentum carried by the quark; $\psi$ is the light-cone Bethe-Salpeter amplitude. The factorization is a good approximation provided the external photon momentum $Q^{2}$ is much larger than all other physical scales. The hard scattering is then evaluated with on-shell quarks carrying neglible $k_{T}$.

The formalism predicts that the cross section for exclusive processes $d \sigma / d t$, where $t$ is the momentum transfer squared, scales like $1 / t^{n-2}$ up to $\operatorname{logs}$, where $n$ is the total number of elementary partons participating in the process. The underlying reason for the power law is scale invariance of the fundamental theory. Further logarithmic dependence is given by QCD scaling violations. In making these assertions one asumes that $t$ is asymptotically large. The dominant contribution to this scattering arises from the valence quark, since every additional parton leads to an additional suppression factor of $1 / t$. Physically the scattering probes the short distance part of the hadron 
wave function. Dominance by the short distance wave functions leads to several predictions such as helicity conservation, color transparency [10, 9] etc.

The successes and failures of this scheme are well known. Calculation of electromagnetic form factors using this factorization scheme has been criticised by several authors [4, 5]. The basic problem is that the momentum scales of the exchanged gluons tend to become rather small, and the applicability of pQCD becomes doubtful. The normalization of form factors is largely unknown; use of asymptotic distribution amplitudes tends to give small normalizations compared to data. Form factor magnitudes can be enhanced by use of model distribution amplitudes [3, 13 which peak closer to the end-points, namely $x \rightarrow 0,1$, which then exacerbates the problem of small internal momentum transfers.

To investigate this problem, there exists an alternate factorization which does not neglect the $k_{T}$ dependence of the hard scattering. The method, which we call "impact parameter factorization", was first used by Botts and Sterman [14 to deal with the Landshoff pinch regions of proton-proton scattering. These regions lie outside the assumptions of the quark-counting formalism, and cannot be described by its factorization scheme. Impact parameter factorization was first applied to electron-beam ( $\gamma^{*}$ initiated) experiments in Ref. [8], in order to accomodate color transparency and nuclear filtering. Li and Sterman [15] developed the method for free-space $\gamma^{*}$ initiated experiments such as the proton's electromagnetic form factor. A consistent feature of this formalism involves attention to the transverse spatial coordinates of quarks and attendant Sudakov effects.

For the case of pion form factor [15] the starting point is,

$F_{\pi}\left(Q^{2}\right)=\int d x_{1} d x_{2} d \vec{k}_{T 1} d \vec{k}_{T 2} \psi^{*}\left(x_{2}, \vec{k}_{T 2}, P_{2}\right) H\left(x_{1}, x_{2}, Q^{2}, \vec{k}_{T 1}, \vec{k}_{T 2}\right) \psi\left(x_{1}, \vec{k}_{T 1}, P_{1}\right)$,

where it is again assumed that the process is factorizable into hard scattering and soft hadronic wave functions $\psi\left(x, \vec{k}_{T}, P\right)$. The calculation is simplified by dropping the $k_{T}$ dependence in the quark propagators in hard scattering kernel $H$, in which case only the combination $\vec{k}_{T 1}+\vec{k}_{T 2}$ appears in the calculation. The authors [15] work in configuration space where this can be 


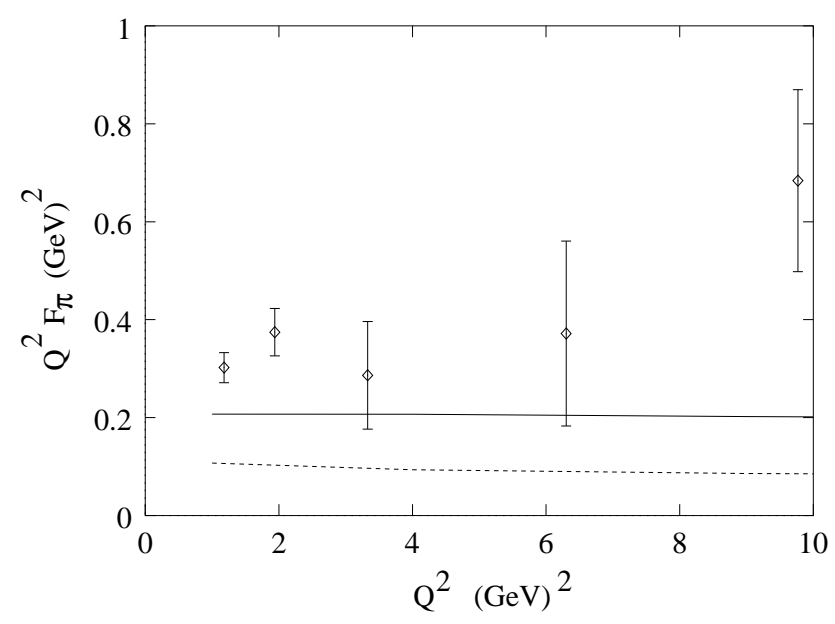

Figure 1: The pion form factor $F_{\pi}\left(Q^{2}\right)$ using the asymptotic (dotted line) and the $C Z$ (solid line) distribution amplitudes. The experimental data with error bars from Ref. [16] are also shown.

written as

$$
F_{\pi}\left(Q^{2}\right)=\int d x_{1} d x_{2} \frac{d^{2} \vec{b}}{(2 \pi)^{2}} \mathcal{P}\left(x_{2}, b, P_{2}, \mu\right) \tilde{H}\left(x_{1}, x_{2}, Q^{2}, \vec{b}, \mu\right) \mathcal{P}\left(x_{1}, b, P_{1}, \mu\right),
$$

where $\mathcal{P}(x, b, P, \mu)$ and $\tilde{H}\left(x_{1}, x_{2}, Q^{2}, \vec{b}, \mu\right)$ are the Fourier transforms of the wave function and hard scattering respectively; $\vec{b}$ is conjugate to $\vec{k}_{T 1}+\vec{k}_{T 2}$, $\mu$ is the renormalization scale and $P_{1}, P_{2}$ are the initial and final momenta of the pion.

Sudakov form factors are obtained by summing the leading and next to leading logarithms using renormalization group (RG) techniques. The wave function at small $b$ is often approximated by the distribution amplitude $\phi(x, 1 / b)$.

The resulting form factor using asymptotic as well as CZ [3] distribution amplitudes is shown in fig. 1. A remarkable fact is that the correct asymptotic $Q^{2}$ behavior is seen beyond the scale of about $Q=1 \mathrm{GeV}$, irrespective of the choice of wave function. In contrast to the Brodsky-Lepage factorization, the $k_{T}$ dependence of the hard scattering is not neglected, and hence this $Q^{2}$ dependence does not follow trivially. Instead, the $Q^{2}$ dependence is a detailed dynamical prediction of the theory, and depends on the relative size 
of intrinsic $k_{T}^{2}$ and $x_{1} x_{2} Q^{2}$. The $Q^{2}$ dependence of the prediction is comparatively robust, since it is weakly dependent on the details of the distribution amplitude.

We note that the normalization of the theoretical result falls below the experimental data for both choices of distribution amplitude. It is good to keep in mind that the distribution amplitudes are not known with much exactitude, so perhaps the models might be improved. Indeed the theoretical normalization of the form factor is comparatively murky, because it strongly depends on such model-dependent details. Moreover, the large difference between theory and experiment at high momenta should be interpreted with caution, since[6] there may be large systematic errors in the experimental extraction of the form factor which are not shown in the figure. Further theoretical issues in this extraction have also been raised in Ref. [17].

Furthermore, the leading order pQCD amplitudes calculated may not give a very reliable estimate of the normalization. Li and Sterman argue that roughly $50 \%$ of the contribution can be regarded as perturbative, since it is obtained from the region where $\alpha_{s} / \pi<0.7$. It may be that higher order contributions in $\alpha_{s}$ are not negligible, and the leading order predictions for the normalization of the form factor cannot be regarded as accurate.

We are left with the following interesting situation: Although the basic Brodsky-Lepage factorization survives at asymptotic $Q^{2}$, the method is sensitive to end-point singularities, and one may need to go to higher orders in $\alpha_{s}$ in order to obtain an accurate prediction for the form factor normalizations. Meanwhile the predicted $Q^{2}$ dependence of the impact parameter factorization appears to be quite robust, and less strongly dependent on the theoretical uncertainties such as the choice of distribution amplitude.

\subsection{The Proton Electromagnetic Form Factor}

The improved impact parameter factorization has also been applied to the proton Dirac form factor $F_{1}^{p}\left(Q^{2}\right)[18]$. The calculation is considerably more complicated compared to the pion due to the presence of three valence quarks. Here also it is necessary to use distribution amplitudes which peak close to the end points if one wishes to fit the experimental normalization of the form factor. The results [18, 19] of the calculation using KS [13] and CZ [3] distribution amplitudes and $c=1$ and 1.14 are shown in fig. 2, where $c$ is a parameter which determines the long and short distance factorization scale. 


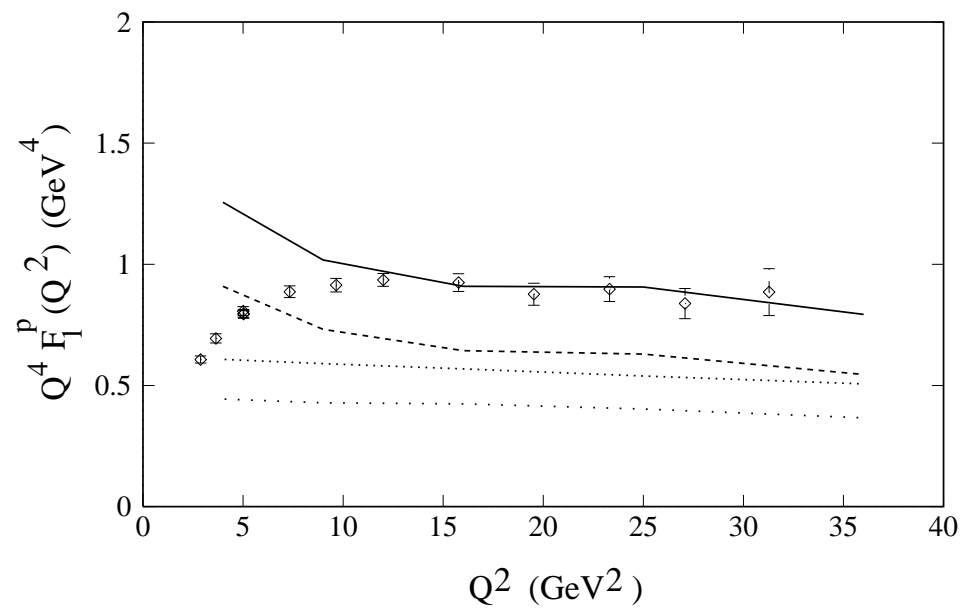

Figure 2: Dependence of proton form factor $Q^{4} F_{1}^{p}$ on $Q^{2}$ using the $K S$ distribution amplitude $(c=1.14$, solid line; $c=1$, dense-dot line) and the $C Z$ distribution amplitude $(c=1.14$, dashed line; $c=1$, dotted line). The experimental data with error bars, taken from Ref. [20], are also shown.

The natural agreement of $Q^{2}$ dependence of the pQCD calculations should be contrasted to data fits obtained using soft overlap models [21, 22]. In such models the $Q^{2}$ dependence depends on the details of the model wave function. Soft overlap model predictions at high momentum have a tendency to fall more strongly than experimental data.

\subsection{Hadron-Hadron Exclusive Processes}

To a rough approximation, the empirically fit power-laws for hadron-hadron exclusive processes tend to agree well with the quark-counting scaling law. However when data is examined in detail, one finds contradictions, such as the violation of helicity conservation selection rules and oscillations [23, 24, 25] around the overall power law momentum dependence of $d \sigma / d t$. It is not commonly appreciated that these signals of processes beyond the quarkcounting model are observed for almost every process tested so far. 


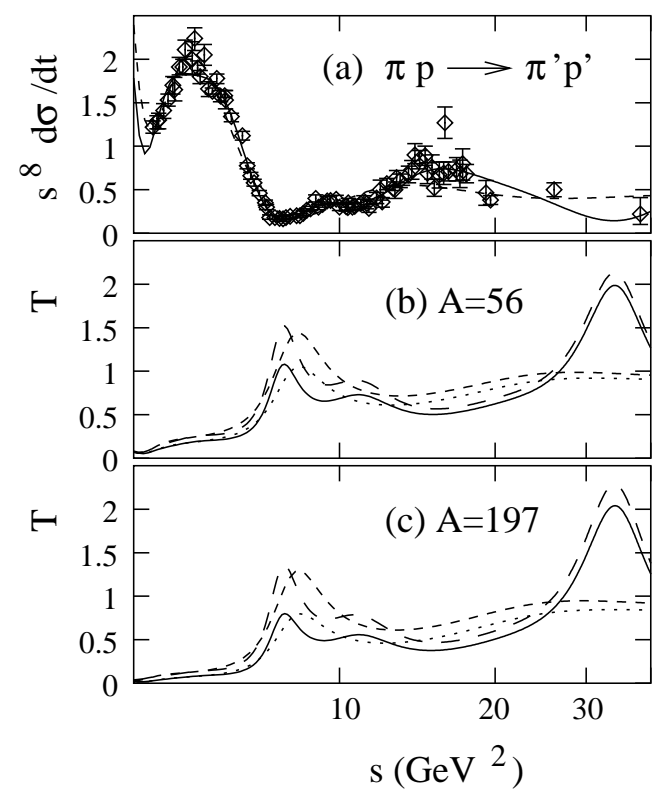

Figure 3: (a) The free space $\pi p 90^{\circ}$ cross section $s^{8} d \sigma / d|t|\left(10^{8}\right.$ GeV $V^{16}$ $\mu \mathrm{b} / \mathrm{GeV}^{2}$ ) using three component model [25] (solid curve) and a two component model (dashed). (b,c) Calculated color transparency ratio for $A=$ 56, 197 using nuclear filtering, in the three component model with $k=10$ (solid), $k=5$ (long dashed) and the two component model with $k=10$ (dotted) and $k=5$ (short dashed). 


\subsection{Free Space Data: $\pi-\mathrm{P}$ to $\pi-\mathrm{P}$ at Fixed Angle}

In Fig. 3a we show results $d \sigma / d t$ for $\pi p \rightarrow \pi^{\prime} p^{\prime}$ scattering at $90^{\circ}$ 25. An overall momentum dependence $|t|^{-8}$ has been factored out of the cross section [26]. The free space cross section is fit in terms of interfering short and long distance amplitudes using a three component (solid curve) and two component model (dashed curve). One amplitude is obtained from the independent scattering diagrams [27], where the quarks scatter at large transverse separations. Leading logarithmic summation calculable in pQCD and related by analyticity to Sudakov effects generates the oscillations [23, 14]. In the three component model we also include a subleading contribution: details are given in Ref. 25]. We note that the oscillations around the overall power dependence are not a small effect. The model incorporating the independent scattering diagrams fits the free space data very well with $\chi^{2} /$ degree of freedom $=1.97$. The Brodsky-Lepage short distance model gives $\chi^{2} /$ degree of freedom $=99$ and is objectively ruled out.

\subsection{Free Space Data: $\gamma-\mathrm{P}$ to $\pi-\mathrm{N}$ at Fixed Angle}

The experimental data 28 for $\gamma p \rightarrow \pi^{+} n$ also shows fluctuations around the overall power behavior as seen in Fig. Ha. This is quite interesting, since in this case the Landshoff pinch amplitudes have been theoretically shown to be subleading at large $Q^{2}$ 229. However at medium $Q^{2}$ even the subleading amplitudes can give significant contributions. We again model the fluctuations in terms of interfering soft and hard contributions. The free space fit is shown in Fig. Фa.

\section{$3 \quad$ Nuclear Targets and Color Transparency}

Color transparency is a natural prediction of pQCD. If exclusive processes at large momentum transfer are actually dominated by short distance, then interaction of hard-struck hadrons with other hadrons is predicted to be small. The hadron interaction cross section $\sigma \propto b^{2}$, where $b$ is the transverse separation between quarks in a color singlet state, vanishes as $b \sim 1 / Q \rightarrow 0$. Experimentally color transparency is measured by observing quasi exclusive 


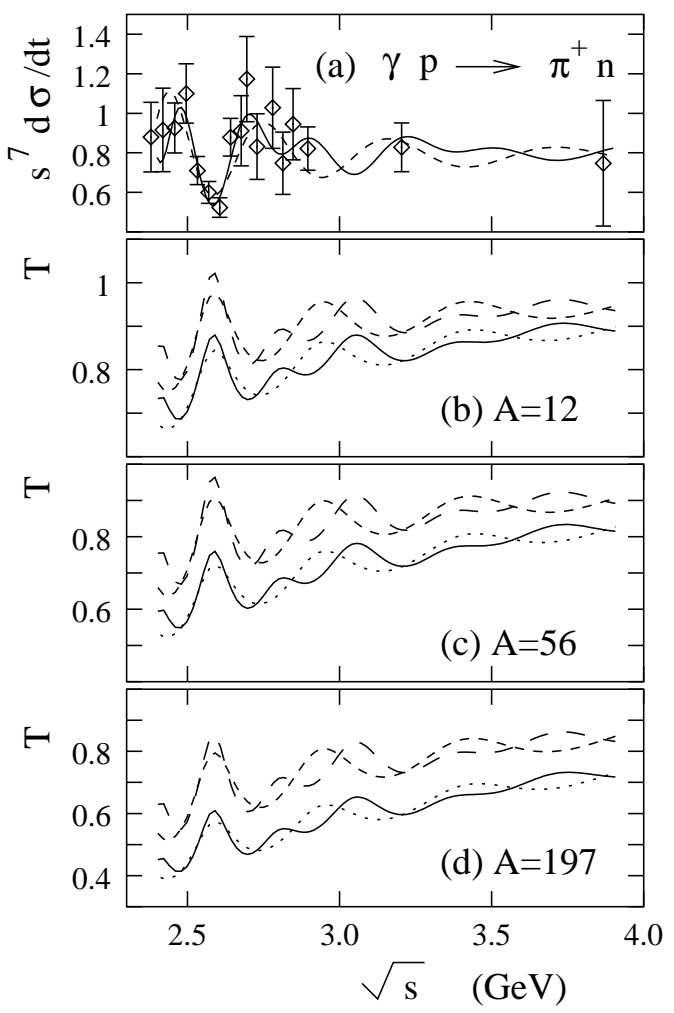

Figure 4: (a) The free space $\gamma p \rightarrow \pi^{+} n 90^{\circ}$ cross section $s^{7} d \sigma / d|t|\left(10^{7}\right.$ GeV $V^{14} n b / G e V^{2}$ ) using three component model (solid curve) and a two component model (dashed). (b,c,d) Calculated color transparency ratio for $A=12,56,197$ using nuclear filtering, in three component model with $k=10$ (solid), $k=5$ (long dashed), and in two component model with $k=10$ (dotted) and $k=5$ (short dashed). 
reactions on nuclear targets such as $e A \rightarrow e^{\prime} p(A-1)$ and comparing it with the corresponding free space process $e p \rightarrow e^{\prime} p^{\prime}$. The experimental results are reported in terms of transparency ratio $T$, defined as

$$
T=\frac{d \sigma_{\text {nuclear }}}{A d \sigma_{\text {free space }}}
$$

At asymptotically large $Q^{2}$, one has asymptotically short distance, and color transparency ratios are unity. This is actually as much as one can say with quark-counting factorization. To say more one must incorporate information about the transverse, or impact parameter separation, hence the use of "impact parameter factorization" 8 .

There are two ways, then, to get to short distance: asymptotically large $Q^{2}$, which we have seen above is not a realistic feature of the laboratory, or by nuclear filtering. The nucleus essentially acts as a transverse-separation filter [8] which preferentially attentuates the soft amplitudes. It follows that the nucleus is a cleaner medium to study exclusive processes than free space: which is quite a surprising prediction.

Several experiments indicate that color transparency [10] and nuclear filtering [7, 8, 30, 31] have been observed at large nuclear number $A$. The first color transparency experiment of Carroll et al [32] convincingly showed that interference effects in proton-proton scattering were filtered away in nuclear targets. Attenuation of the Landshoff-induced oscillations, seen in free-space [23], in the nucleus reproduces the oscillating transparency [0]. In contrast, other models based on a classically expanding cross section [33. do not fit the data. Extraction of the attenuation cross section in nuclear targets show values significantly below the Glauber theory values [9]. The FNAL E-665 experiment [34] also proved consistent with filtering effects [35].

Electron beam experiments remain controversial, with few signals of interesting $Q^{2}$ dependence [36]. A basic feature of $\gamma^{*}$-initiated reactions is that most events are knocked out from the back side of the nucleus. The resolving power of such experiments to measure the size of propagating states is rather modest, unless one has very high experimetal precision. The $A$ dependence is a particularly useful tool [9] to measure effective attenuation cross sections. O'Neill et al [37] showed that effective attenuation cross sections extracted from $A\left(e, e^{\prime} p\right)$ SLAC data were smaller than Glauber theory calculations by a statistically significant amount. However, the precision of the data [36] was insufficient to establish a large effect, and model dependence in the choice 


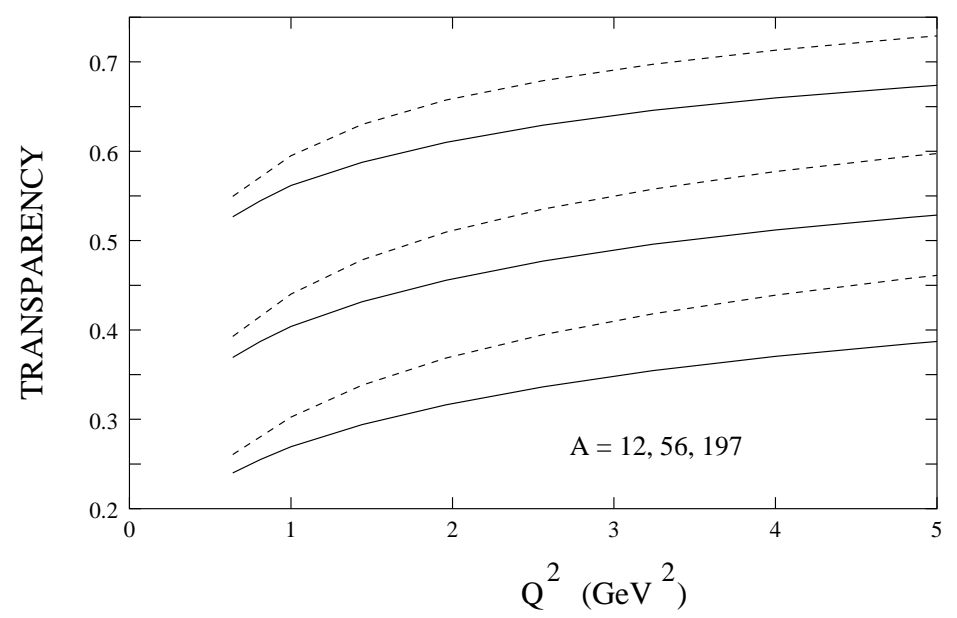

Figure 5: The calculated pion transparency ratio for different nuclei as a function of $Q^{2}$. The solid and dashed curves use the CZ and asymptotic distribution amplitudes respectively and correspond to $A=12,56$ and 197 from top to bottom. More details are given in Ref. [38].

of the normalization of hard scattering is another complication. Reports on new $\left(e, e^{\prime} p\right)$ beam experiments from CEBAF are expected shortly.

\subsection{New Color Transparency Calculations}

We have recently calculated color transparency ratio for several processes which include $\pi A \rightarrow \pi^{\prime} p(A-1), \gamma A \rightarrow \pi N(A-1), e A \rightarrow e^{\prime} p(A-1)$ and $e A \rightarrow e^{\prime} \pi A$. For the case of $e A \rightarrow e^{\prime} p(A-1)$ and $e A \rightarrow e^{\prime} \pi A$ we did detailed impact-parameter calculations using leading order pQCD including the Sudakov effects. The more complicated processes $\pi A \rightarrow \pi^{\prime} p(A-1)$, $\gamma A \rightarrow \pi N(A-1)$ were modelled in terms of interfering long and short distance amplitudes following the earlier work on proton proton quasi elastic scattering in nuclear medium [7, 30]. The magnitude of short and long distance amplitudes were obtained phenomenologically by making a fit to the corresponding free space process.

In Fig. 5 we show the $Q^{2}$ dependence of the transparency ratio for electroproduction of pions using the $\mathrm{CZ}$ and asymptotic distribution amplitudes [38]. The scale of $Q^{2}$ ranging up to $5 \mathrm{GeV}^{2}$ may benefit from explanation. At 


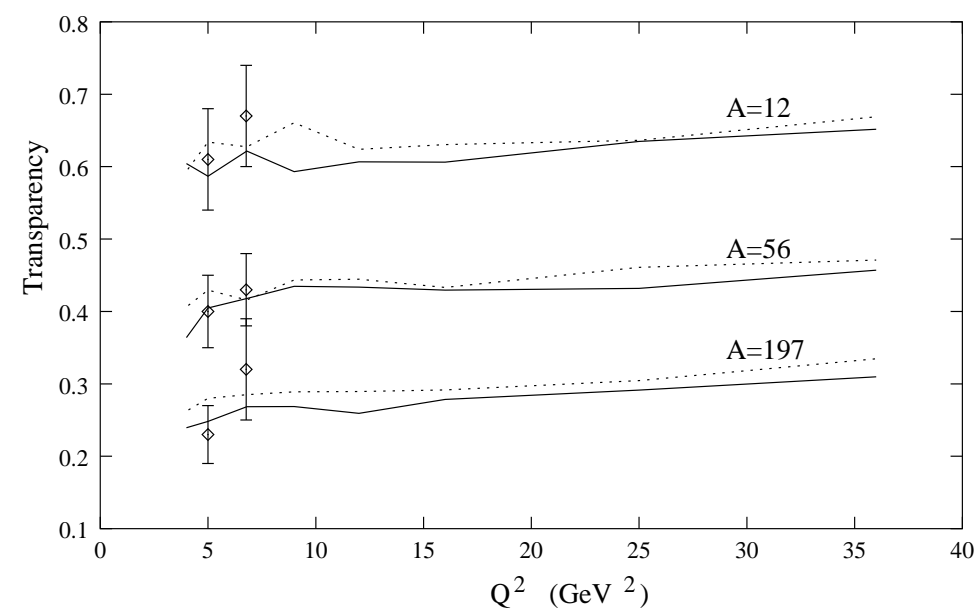

Figure 6: Predicted transparency ratio [38] for the proton for different nuclei using the KS end-point dominated model for distribution amplitude. The experimental points are taken from Ref. [26,27]. The solid curves are calculated with $k=10$ and the dashed curves with $k=9$.

the exclusive production point, the relativistic boost factor of a pion is given by $\gamma=Q^{2} /\left(2 m_{\pi}^{2}\right) \sim 25\left(Q^{2} / \mathrm{GeV}^{2}\right)$. Since even a $1 \mathrm{GeV}$ pion is highly relativistic, we may suppose that the perturbative calculations may well apply in the comparatively small $Q^{2}$ regime. These calculations show a rather striking rise with $Q^{2}$ of the transparency ratio, which should be easily observable experimentally. The fact of a rise does not depend much on the distribution amplitude, but the slope of the rise does: we discuss the reasons shortly when we review the proton. For these calculations we used $\Lambda_{Q C D}=200 \mathrm{MeV}$. We adjusted the value of the parameter $k$, defined by $\sigma_{\text {attenuation }}=k b^{2}$, so that the predicted results for proton (discussed later) are in agreement with the SLAC data [36, 37. This selects the value of $k$ to be approximately equal to 10.

Results for the $Q^{2}$ dependence of the proton transparency ratio [38] from various models are shown in Figs. (6, 7). A standard model for the distribution amplitude, the KS model, was used to generate Fig. 6. One sees that the calculation with the KS model has a rather flat $Q^{2}$ dependence. At first this result was surprising, assuming short-distance dominance and the expectation of a rapidly increasing function of $Q^{2}$, but in retrospect the result appears quite natural. As in the earlier discussion of the pion, these results 


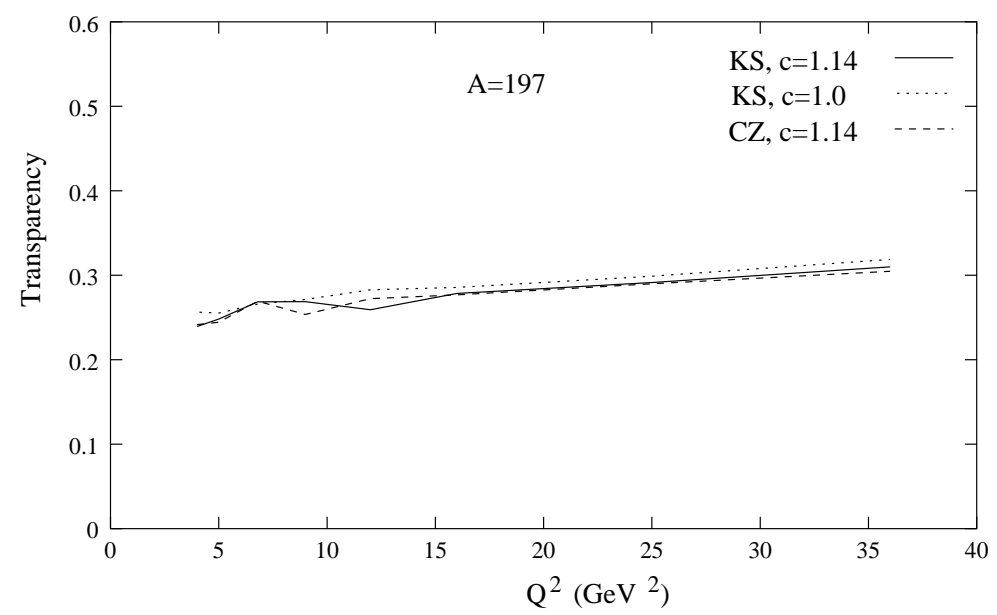

Figure 7: The sensitivity of the calculated transparency ratio to different proton distribution amplitudes and the factorization scale parameter $c$. The solid, dotted and dashed curves correspond to the KS wavefunction with $c=1.14, K S$ distribution amplitude with $c=1.0$ and the $C Z$ distribution amplitude with $c=1.14$ respectively. All calculations use $A=197$. More details are given in Ref. [38]

depend on the distribution amplitude model, which can be categorized into two types. The KS model is an end-point dominated distribution amplitude, which is known to produce its dominant contributions from long-distance components of the quark wave functions. For this reason use of the KS wave function in the free-space form factor has led to many questions of theoretical consistency, mentioned earlier. Precisely the same lack of a dominant short-distance contribution is responsible for the calculated flat dependence on $Q^{2}$. Turning to Fig. 7, which compares the CZ and KS models, both of which are end-point dominated, one sees nearly identical flat $Q^{2}$ behavior. This indicates that the details of the model do not matter so long as they are end-point models. The figure also shows the dependence on the factorization scale parameter $c$. Rather interestingly, a substantial dependence on $c$ of form factors in free space drops out in the transparency ratio.

If the transparency ratio is flat with $Q^{2}$, does it mean that color transparency has not been observed? No, because important contributions to the free-space denominator are being filtered away by a large nucleus, depleting 


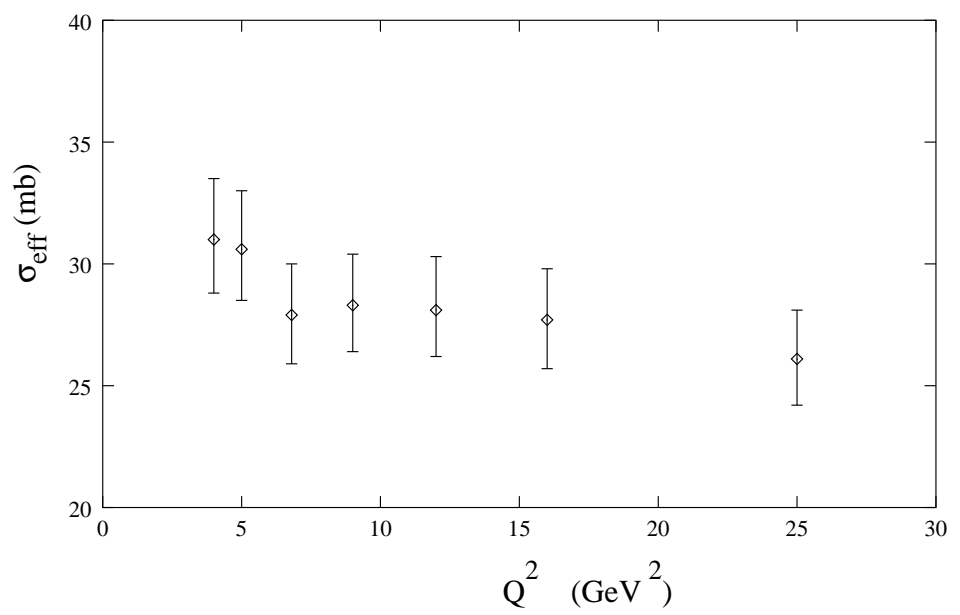

Figure 8: The proton attenuation cross section $\sigma_{\mathrm{eff}}$ as a function of $Q^{2}$ extracted from the monte carlo calculations. The extracted value is significantly smaller than the standard Glauber theory value of $40 \mathrm{mb}$.

the numerator, making the interpretation of the $Q^{2}$ dependence ambiguous [30]. Fortunately experiments also have the $A$ dependence, which can be expressed as an empirically defined effective attentuation cross section $\sigma_{\text {eff }}$. In the standard Glauber theory of $p A$ scattering this quantity is flat with energy in the region of interest and has a value of about $40 \mathrm{mb}$. Observation of $\sigma_{\text {eff }}$ substantially below the Glauber value would be a signal of color transparency. We evaluated our predictions as if they were experimental data, extracted $\sigma_{\text {eff }}$, and found the value to be about $31 \mathrm{mb}$ at $Q^{2}=4 \mathrm{GeV}^{2}$ and slowly decreasing with energy as shown in fig. 8. This value is substantially below the Glauber value: so color transparency can be observed in $e, e^{\prime} p$ measurements of sufficiently high precision.

Finally in fig. 3 $\mathrm{b}, \mathrm{c}$ we show results for the transparency ratio for the process $\pi A \rightarrow \pi^{\prime} p(A-1)$. Rather interesting oscillations are predicted for the transparency ratio. These predictions can be tested in future experiments. The results for the transparency ratio for $\gamma A \rightarrow \pi^{+} n(A-1)$ are shown in Fig Gb,c,d. We again predict fluctuations in the transparency ratio which can be tested in the near future at CEBAF [39].

Acknowledgements: Work was partially supported by DOE grant number 85ER40214. 


\section{References}

[1] S. J. Brodsky and G. R. Farrar, Phys. Rev. D 11, 1309 (1975).

[2] S. J. Brodsky and G. P. Lepage, Phys. Rev. D 24, 2848 (1981).

[3] V. L. Chernyak and A. R. Zitnitsky, Phys. Rep. 112, 173 (1984); Nucl. Phys. B 246, 52 (1984).

[4] N. Isgur and C. Llewelyn-Smith, Phys. Rev. Lett. 52, 1080 ,(1984.)

[5] A. V. Radyushkin, Acta Phys. Polonica, B15, 403 (1984); A. P. Bakulev and A. V. Radyushkin, Phys. Lett. B 271, 223 (1991).

[6] G. Sterman and P. Stoler, hep-ph/9708370, Annual Review of Nuclear and Particle Science 47, (1997) 193.

[7] J. P. Ralston and B. Pire, Phys. Rev. Lett. 61, 1823 (1988).

[8] J. P. Ralston and B. Pire, Phys. Rev. Lett. 65, 2343 (1990).

[9] P. Jain, B. Pire and J. P. Ralston, Phys. Rep. 271, 67 (1996).

[10] S. J. Brodsky, in Proc. 13th Int. Symp. on Multiparticle Dynamics, Vollendam, 1982, eds. W. Kittel et al. (World Scientific, Singapore, 1982); A. H. Mueller, in Proc. Rencontres de Moriond Les Arcs, France, 1982, ed. J. Tran Thanh Van (Editions Frontiers, Gif-sur Yvette, 1982); S. J. Brodsky and A. H. Mueller, Phys. Lett. B 206 685, (1988).

[11] G. R. Farrar and D. R. Jackson, Phys. Rev. Lett. 43 (1979) 246.

[12] A. V. Efremov and A. V. Radyushkin, Phys. Lett. B 94 (1980) 245.

[13] I.D. King and C.T. Sachrajda, Nucl. Phys. B 279 (1987) 785.

[14] J. Botts and G. Sterman, Nucl. Phys. B 325 (1989) 62.

[15] H.-N. Li and G. Sterman, Nucl. Phys. B 381, 129 (1992).

[16] C. J. Bebek et al., Phys. Rev. D 17 (1978) 1693.

[17] C. E. Carlson and J. Milana, Phys. Rev. Lett. 65, (1979) 246. 
[18] H.-N. Li Phys. Rev. D 48, 4243 (1993).

[19] B. Kundu, H.-N. Li, J. Samuelsson and P. Jain, hep-ph/9806419, Euro. Phys. Journal C 8, Vol 4, 637 (1999).

[20] G. Arnold et al., Phys. Rev. Lett. 57, 174 (1986).

[21] A. V. Radyushkin, Phys.Rev. D 58 (1998) 114008, hep-ph/9803316.

[22] M. Diehl, Th. Feldman, R. Jacob and P. Kroll, hep-ph/9811253, Eur. Phys. J. C 8 (1999) 409.

[23] B. Pire and J. P. Ralston, Phys. Lett. B 117, 233 (1982); J. P. Ralston and B. Pire, Phys. Rev. Lett. 49, 1605 (1982); A. Sen, Phys. Rev. D 28, 860 (1983).

[24] C. Carlson, V. Chashkuhnashvilli and F. Myhrer, Phys. Rev. D 46, 2891 (1992); P. Ramsey and D. Sivers, Phys. Rev. D 52, 116, (1995).

[25] P. Jain, B. Kundu and J. P. Ralston, hep-ph/0005126

[26] D. P. Owen et al., Phys. Rev. 181, 1794 (1969); K. A. Jenkins et al., Phys. Rev. D 21, 2445 (1980); C. Baglin et al., Nucl. Phys. B 216, 1 (1983); J. Blazey, Ph. D. thesis, University of Minnesota (1986).

[27] P. V. Landshoff, Phys. Rev. D 10, 1024 (1974).

[28] R. L. Anderson et al., Phys. Rev. D 14, 679 (1976).

[29] G. Farrar, G. Sterman, and H. Zhang, Phys. Rev. Lett. 62, 2229 (1989).

[30] P. Jain and J. P. Ralston, Phys. Rev. D 48 1104, (1993).

[31] S. J. Brodsky and G. F. de Teramond, Phys. Rev. Lett. 60, 1924 (1988).

[32] A. S. Carroll et. al., Phys. Rev. Lett. 61, 1698 (1988).

[33] G. R. Farrar, H. Liu, L. L. Frankfurt, and M. I. Strikman, Phys. Rev. Lett. 61, 686 (1988).

[34] M. R. Adams et al, Phys. Rev. Lett. 74, 1525 (1995). 
[35] B. Z. Kopeliovich, J. Nemchick, N. N. Nikolaev, and B. G. Zakharov, Phys. Lett. B 309, 179 (1993); Phys. Lett. B 324, 469 (1994).

[36] N. Makins et. al, Phys. Rev. D 12, 163 (1994).

[37] T. G. O ' Neill et. al, Phys. Lett. B 351, 87 (1995).

[38] B. Kundu, J. Samuelsson, P. Jain and J. P. Ralston, hep-ph/9812506, Physical Review D 62 (2000) 113009.

[39] H. Gao, R. J. Holt, Jefferson Lab experiment E94-104. 